\title{
Study on Distribution Characteristics and Displacement Mechanism of Microscopic Residual Oil in Heterogeneous Low Permeability Reservoirs
}

\author{
Yujia Fang $\mathbb{D}^{1}$, Erlong Yang $\mathbb{D}^{1}{ }^{1}$ and Xiaona Cui $\mathbb{D}^{1,2}$ \\ ${ }^{1}$ Key Lab of Ministry of Education for Enhancing the Oil and Gas Recovery Ratio, Department of Petroleum Engineering, \\ Northeast Petroleum University, Daqing 163318, China \\ ${ }^{2}$ Harold Vance Department of Petroleum Engineering, Texas A\&M University, College Station, TX 77843, USA
}

Correspondence should be addressed to Erlong Yang; openmind_aa@sina.com

Received 12 June 2019; Revised 20 August 2019; Accepted 6 September 2019; Published 21 October 2019

Academic Editor: Jaewon Jang

Copyright (c) 2019 Yujia Fang et al. This is an open access article distributed under the Creative Commons Attribution License, which permits unrestricted use, distribution, and reproduction in any medium, provided the original work is properly cited.

\begin{abstract}
In order to explore the development methods suitable for heterogeneous low permeability reservoirs and study the distribution characteristics of residual oil, photoetched glass and artificial core models with three permeability ratios of 1, 6, and 9 were prepared in this research. Three displacement schemes including polymeric surfactant flooding, polymeric surfactant with binary flooding, and binary flooding were designed at the same expenses to obtain the displacement mechanism of various residual oil saturations. The results show that the best displacement efficiency can be achieved by polymeric surfactant flooding, followed by polymeric surfactant with binary flooding, and binary flooding for the models with the same permeability ratio. Binary flooding mainly activates cluster and oil drop residual oils, polymeric surfactant with binary flooding mainly activates cluster, oil film, and column residual oils, whereas polymeric surfactant flooding mainly activates cluster, oil drop, and column residual oils. In addition, with the increase of the model permeability ratio, the recovery ratio of water flooding decreases, whereas the enhanced oil recovery and the variations in residual oil saturation gradually increase after carrying out different displacement measures. The viscoelastic and shearing effects of the polymeric surfactant flooding system can better displace the residual oil, assisting in the further development of heterogeneous low permeability reservoirs.
\end{abstract}

\section{Introduction}

Due to the complex pore structure and severe heterogeneity of low permeability reservoirs, causing large volumes of residual oil in the pores, it is imperative to take reasonable measures to reduce the effect of reservoir heterogeneity on ultimate recovery. Numerous technologies for improved oil recovery have been developed to date [1-5]. Polymer flooding is widely used for improved recovery efficiency in China's oilfield development [6-9]. Concerning the mechanism of polymer flooding, the viscoelastic characteristic of the polymer solution can improve oil displacement efficiency as the residual oil is pulled out by the polymer solution. The larger the viscoelasticity of the polymer, the more the reduction in the residual oil saturation [10]. On the other hand, injecting polymer into the pores will easily cause profile reversal after water flooding [11]. In addition, polymer retention in the pores increases the cost of oilfield development, as most of the residual oil remains in the reservoir [12-16]. For the ternary flooding system, the polymer in the ternary system improves the oil-water mobility ratio and acts to block the high permeability layer, thereby expanding the sweep volume. On the other hand, the surfactant in the ternary system can reduce the interfacial tension between the oil and water, making the oil-water interface film more susceptible to deformation, and the residual oil is gradually broken into small oil droplets and dragged towards the flow direction [17]. However, permeability reduction and flow problems occurred during the oil displacement process, due to the reaction between alkalis (such as $\mathrm{NaOH}$ and $\mathrm{Na}_{2} \mathrm{CO}_{3}$ ) and divalent cation $\left(\mathrm{Ca}^{2+}, \mathrm{Mg}^{2+}\right)[18,19]$. Polymeric surfactant 


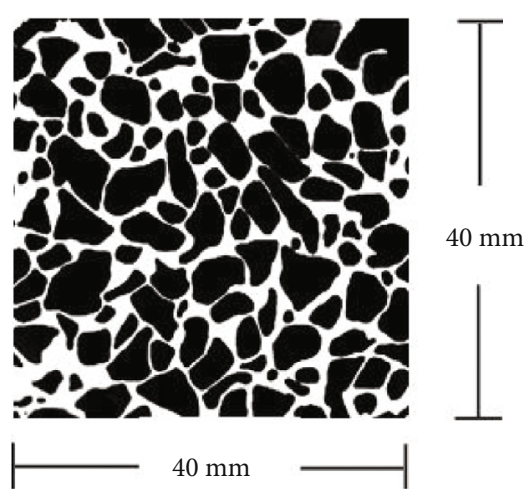

(a)

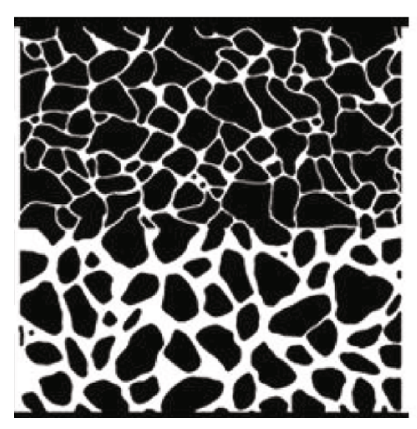

(b)
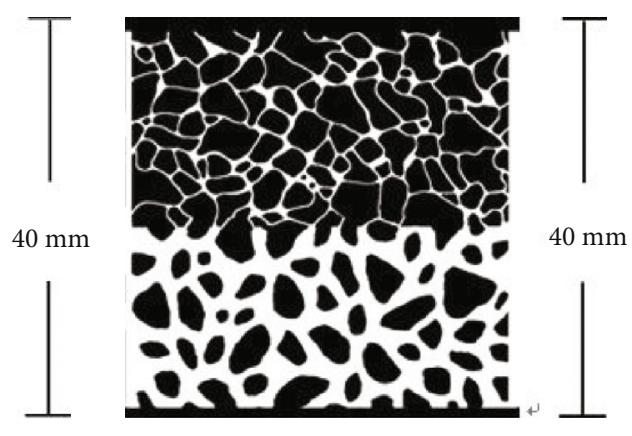

(c)

FIGURE 1: Schematic diagram of planar heterogeneous photolithographic glass model: (a) the permeability ratio of 1; (b) the permeability ratio of 6 ; (c) the permeability ratio of 9.

(also called active polymer) is a new type of oil displacement agent recently developed and put into field application, which is a group with special functions grafted on the molecular chain of the polymer. Through interactions within and between molecular chains, it realizes the functions of emulsification, viscosity increase, oil washing, and water plugging [20-24]. The main mechanism of enhanced oil recovery by polymeric surfactant flooding is to improve oil displacement efficiency by emulsification while realizing mobility control.

Very few studies on visual displacement experiments are carried out for low permeability reservoirs and quantitative analysis of different types of residual oil saturation; therefore, the mechanism that leads to low recovery ratio at the micro level is not clear. Oil displacement experiment using a photoetching glass model can visually see the deformation, migration, and accumulation of fluid in the pore structure; the distribution of different types of residual oil is different when different displacement fluids are injected. Yue et al. studied the effect of polymer particles on the saturation of residual oil, dividing the residual oil into clusters, columns, membranes, and blind ends. Polymer particle flooding can reduce the saturation of cluster, column, and membrane residual oil [25]. Wu et al. studied different production strategies by water flooding experiments with an etched-glass micromodel. It was found that altering the injection direction is a more effective method of enhancing oil recovery [26].

Generally, there is little research on the pore structure that affects the recovery rate of low permeability oil layers at home and abroad. In addition, the internal relationship between pore structure and fluid distribution from a microscopic perspective is seldom analyzed. Despite the fact that most reservoirs have entered high water cut stage, the distribution of residual oil is still unclear. Therefore, it is crucial to study the root causes that affect the fluid distribution and the formation of residual oil so as to find effective methods to improve the recovery rate of low permeability reservoirs. The low-resistance polymeric surfactant to improve displacement effect for low permeability reservoirs has not been used by anyone in previous investigations. This paper uses the photoetching glass model and artificial cores with different permeability ratios to simulate the planar heterogeneous reservoir. In order to explore the adaptability of polymeric surfactant in low permeability reservoirs, different displacement schemes with the same expenses were designed. In addition, the influence of heterogeneity on the distribution of residual oil was obtained by analyzing the variation in the values of residual oil saturation. The above research can provide theoretical support for the development of low permeability reservoirs.

\section{Experimental Design}

2.1. Experimental Material. The cores were obtained from the Daqing Oilfield; planar heterogeneous photolithographic glass models were made with different permeability ratios of 1,6 , and 9 , where permeability ratio is defined as the ratio of the maximum permeability to the minimum permeability of the model. The average permeability of the models is $25 \times 10^{-3} \mu \mathrm{m}^{2}$, which is measured by the method of Fang et al. [27], and the dimensions are $40 \mathrm{~mm} \times 40 \mathrm{~mm}$. The pore structure photos of a natural core casting thin section were taken with a permeability ratio of 1 . Photochemical etching technique was used to obtain the photoetched glass model which is basically consistent with the actual oil layer. When making the glass model with a permeability ratio 6, two Daqing cores with a permeability ratio of 6 were selected and photos were taken. The two sets of photos are spliced and placed on a glass plate; after exposure, development, and high temperature calcination, a glass model can be obtained. Using the same method, the glass model with a permeability ratio of 9 can be obtained. The wettability of the glass model is designed to be hydrophilic according to the selected core characteristics, and the porosity and groove's depth of the glass models are $20.1 \%$ and $0.45 \mathrm{~mm}$, respectively. In addition, the grain size of the model is $1-3 \Phi$; pore size and throat size of the glass models are $10-100 \mu \mathrm{m}$ and $1-10 \mu \mathrm{m}$, respectively; and pore volume of the glass models is $145 \mu \mathrm{L}$. The schematic diagram of the glass model is shown in Figure 1. An artificial flat core model with dimensions of $30 \mathrm{~mm} \times 30 \mathrm{~mm} \times 4.5 \mathrm{~mm}$ and permeability ratios of 1,6 , and 9 , respectively, was utilized. Simulated oil used from the Daqing oilfield, with a viscosity of $10 \mathrm{mPa} \cdot \mathrm{s}$ at $45^{\circ} \mathrm{C}$ and water with mineralization of $508 \mathrm{mg} / \mathrm{L}$, was used for microscopic water flooding 
experiments. The composite ionized water with the mineral concentration of $3700 \mathrm{mg} / \mathrm{L}$ was used to prepare the polymer solution. The polyacrylamide (HPAM) produced by Daqing has relative molecular weight of $1700 \times 10^{4}$ with hydrolysis degree of $26.7 \%$, and carboxybetaine amphoteric surfactant with effective content of $25 \%$. A new type of chemical oil displacement agent, the polymeric surfactant "Haibo Type III" produced by Shanghai Haibo Company, with a solid content of $88 \%$ was used.

2.2. Experimental Apparatus. The aggregation morphology of the chemical agent was measured by a FEI Quanta 450 cold field emission scanning electron microscope in the United States. The samples were prepared at a temperature of $-196^{\circ} \mathrm{C}$. Micro displacement experimental equipment includes image analysis system, video recording system, microscope camera, light power, and microscopic pump. The flow charts of the artificial core and glass model experiments are shown in Figures 2 and 3.

2.3. Experimental Scheme. Three kinds of oil displacement systems were designed with the same price. The specific schemes are as follows: water flooding at a constant rate of $0.03 \mathrm{~mL} / \mathrm{h}$ to a water cut of $98 \%$.

(1) Inject 0.68 PV polymeric surfactant $(\mathrm{Cp}=600 \mathrm{mg} / \mathrm{L})$ with the viscosity of $14.75 \mathrm{mPa} \cdot \mathrm{s}$ and then initiate water flooding until the water cut reached $98 \%$

(2) Inject $0.17 \mathrm{PV}$ polymer/surfactant solution with the viscosity of $9.2 \mathrm{mPa} \cdot \mathrm{s}$ (the concentration of the polymer is $1000 \mathrm{mg} / \mathrm{L}$, the concentration of surfactant is $0.1 \%$ ); afterwards, initiate water flooding until the water cut reached $98 \%$

(3) Inject $0.1 \mathrm{PV}$ polymeric surfactant $(\mathrm{Cp}=600 \mathrm{mg} / \mathrm{L})$, then inject $0.15 \mathrm{PV}$ polymer/surfactant solution (the concentration of the polymer is $1000 \mathrm{mg} / \mathrm{L}$ and the concentration of the surfactant is $0.1 \%$ ); finally, initiate water flooding until the water cut reached $98 \%$

2.4. Experimental Process. Oil displacement experiment of the photoetching glass model consists of the following processes: (1) saturate with oil after vacuuming the photoetching glass model, (2) water flooding at a constant rate of $0.03 \mathrm{~mL} / \mathrm{h}$ until the end of oil production from the model, (3) inject the three displacement systems at a constant speed of $0.03 \mathrm{~mL} / \mathrm{h}$ and observe the distribution of residual oil in the model, (4) calculate the oil displacement efficiency and all kinds of residual oil saturation after the experiment, and (5) replace the model and repeat the above steps.

The artificial flat core flooding experiment consists of the following processes: (1) saturate with formation water after the core is vacuumed, then inject the simulated oil into the model until the outlet end is free of water; afterwards, initiate water flooding until there is no more oil production from the core; (2) inject three displacement systems and stop the displacement process when the water cut reaches $98 \%$; (3) replace the model and repeat the above steps.

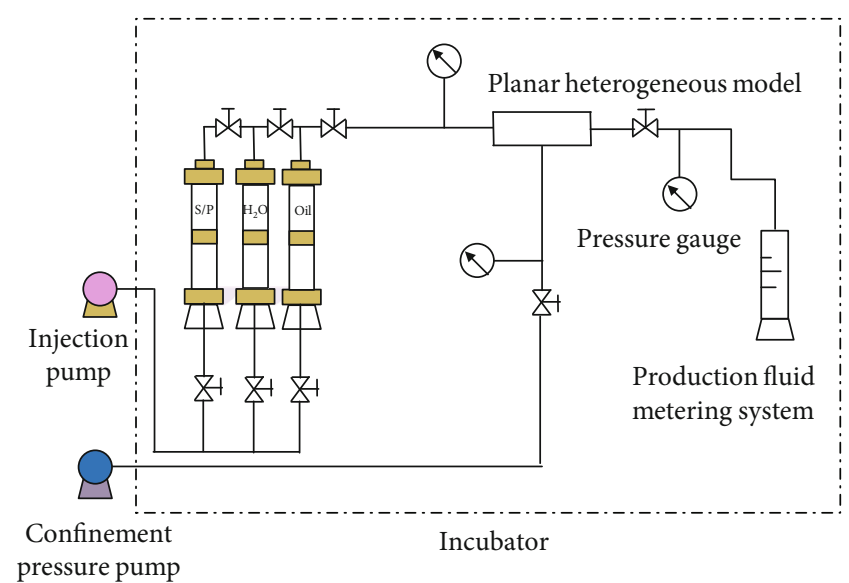

FIgURE 2: Flow chart of the heterogeneous artificial core experiment.

2.5. Saturation Measurement of Various Residual Oils. The displacement images were read through a function in MATLAB. Water, oil, and glass phases were distinguished by giving grey thresholds. After carrying out oil displacement, the residual oil saturation is equal to the area ratio of oil to pore space [28]. In order to calculate a certain type of residual oil saturation, the total number of pixels of the residual oil block was counted to obtain the total area of the oil block. Different types of residual oil saturations were obtained by taking the ratio of the oil block area to the pore area.

\section{Experimental Results and Analysis}

3.1. The Distribution of Micro Residual Oil after Different Flooding Systems. Through the video recording system, the distribution characteristics of microscopic residual oil can be photographed in real time. Residual oil can be divided into 5 types according to its cause of formation and distribution in pores including cluster, columnar, oil drop, blind end, and oil film $[26,27]$. Taking polymeric surfactant flooding as an example, the distribution of residual oil under polymeric surfactant flooding with different permeability ratios can be obtained as shown in Figures 4-6. By processing the experimental images, the recovery ratio and the values of residual oil saturation after different displacement flooding systems can be obtained; the displacement results are shown in Figures 7 and 8 (polymer solution is replaced by P, binary flooding is replaced by $\mathrm{S} / \mathrm{P}$, and polymeric surfactant flooding is replaced by PS). Considering the effect of the systematic error of experimental technology, operating error, and data processing error on experimental results, each experiment with different oil displacement measures was repeated 5 times; thus, the mean value of the displacement efficiency and saturation of various residual oils was obtained. Using the relative uncertainty calculation formula (equation (1)), the relative uncertainty of each group can be obtained [29]. It can be seen from Figures 7 and 8 that the relative uncertainty of each group is within $\pm 5 \%$, so the reliability of the experimental results can be proved. With the increase of the permeability ratio, the displacement efficiency of water flooding gradually decreases, because the larger the permeability 


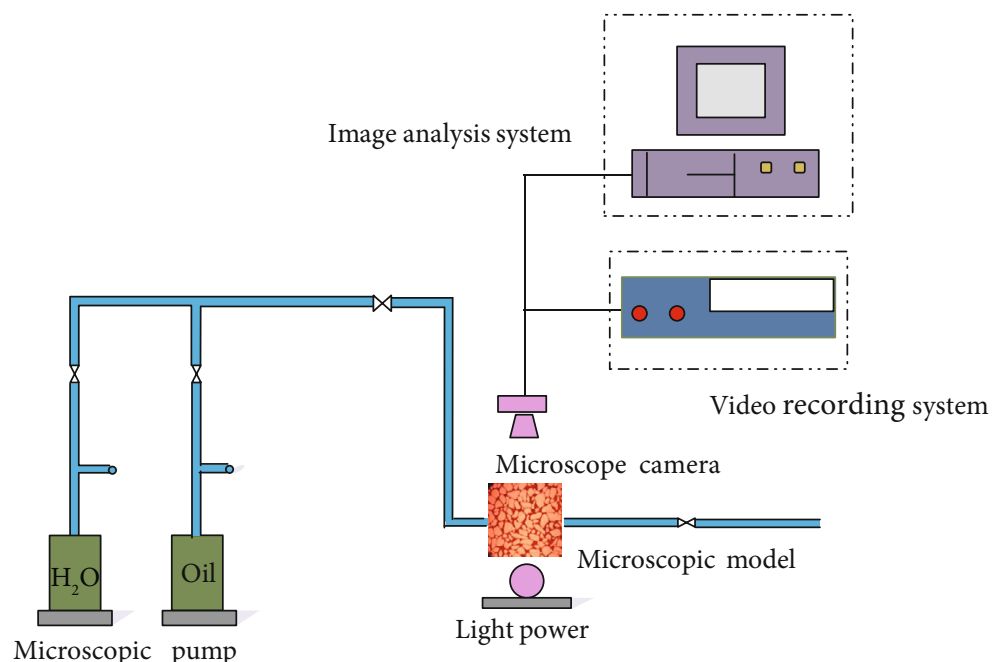

FIGURE 3: Flow chart of the glass model experiment.

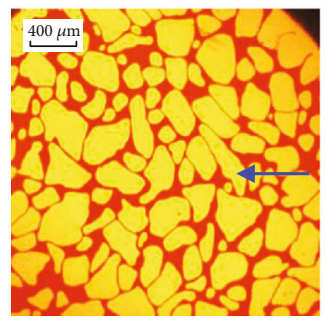

$0 \mathrm{PV}$

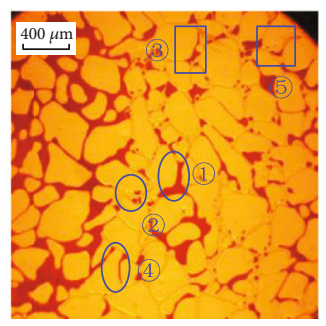

$0.3 \mathrm{PV}$

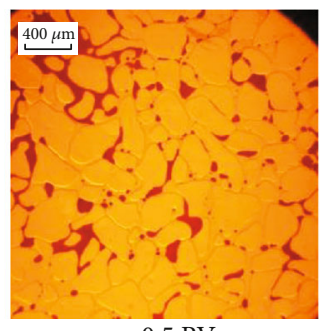

$0.5 \mathrm{PV}$

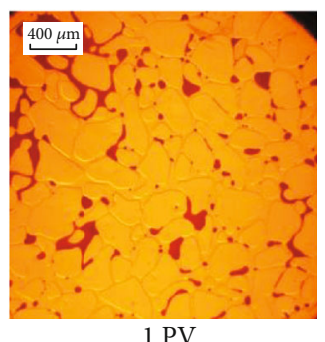

$1 \mathrm{PV}$

FIgURE 4: Distribution of residual oil under polymer surfactant flooding with a permeability ratio of 1 (1) cluster, (2) oil drop, (3) oil film, and (4) columnar; the red part represents oil; the injection direction of the displacement agents is from right to left).

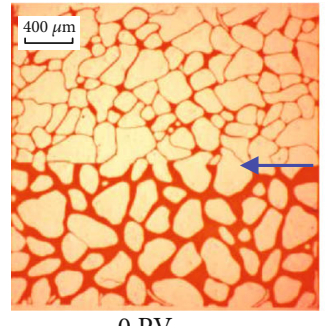

$0 \mathrm{PV}$

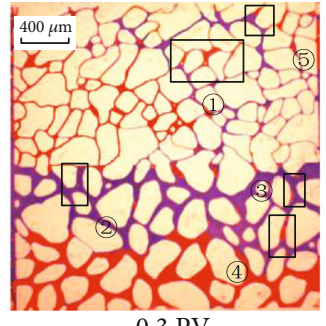

$0.3 \mathrm{PV}$

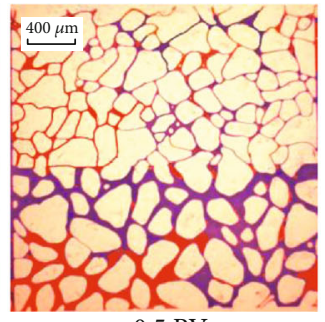

$0.5 \mathrm{PV}$

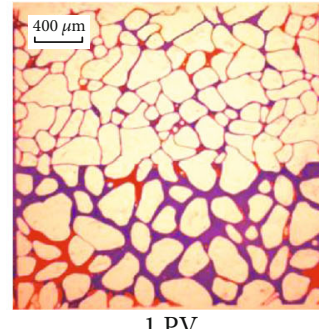

$1 \mathrm{PV}$

Figure 5: Distribution of residual oil under polymer surfactant flooding with a permeability ratio of 6 (1) cluster, (2) oil drop, (3) oil film, (4) columnar, and (5) blind end; the red part represents oil; the blue part represents the displacement fluid; the injection direction of the displacement agents is from right to left).

ratio, the greater the difference of pores and throats between high and low permeability regions, resulting in the formation of the dominant flowing path in high permeability areas. In addition, the saturation of cluster residual oil gradually increases, while the saturation of other residual oil gradually decreases.

$$
u=S(\bar{x})=\sqrt{\frac{\sum_{i=1}^{n}\left(x_{i}-\bar{x}\right)}{n(n-1)}}, \quad U_{r}=\frac{u}{\bar{x}}
$$

After the implementation of the different oil displacement schemes, on the basis of conventional water flooding, polymeric surfactant flooding has shown better oil displacement effect, followed by polymeric surfactant with subsequent binary flooding and binary flooding. When the permeability ratio is 1 , recovery rate is increased by $13.92 \%, 10.20 \%$, and $8.72 \%$, respectively; when the ratio is 6 , recovery rate is increased by $15.15 \%, 11.30 \%$, and $9.71 \%$, respectively; and when the ratio is 9 , recovery rate is increased by $17.51 \%, 13.78 \%$, and $11.90 \%$, respectively. The viscosity of the low-resistance polymeric surfactant is 


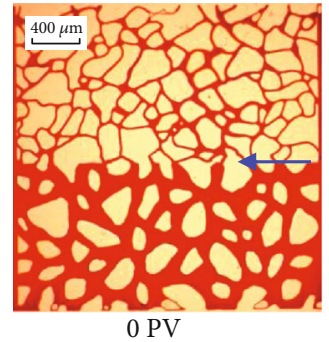

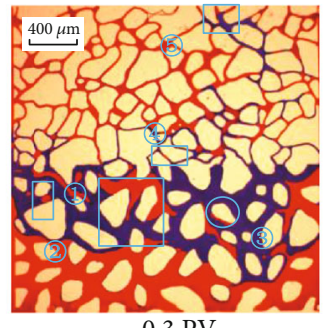

$0.3 \mathrm{PV}$

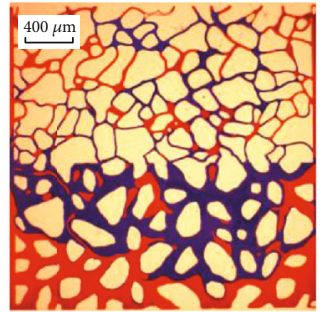

$0.5 \mathrm{PV}$

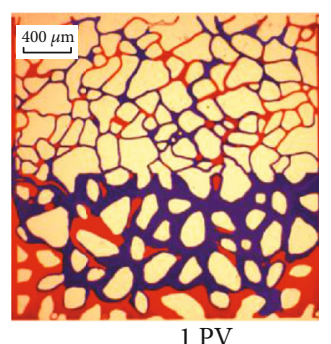

$1 \mathrm{PV}$

FIGURE 6: Distribution of residual oil under polymer surfactant flooding with a permeability ratio of 9 (1) cluster, (2) oil drop, (3) oil film, (4) columnar, and (5) blind end; the red part represents oil; the blue part represents the displacement fluid; the injection direction of the displacement agents is from right to left).

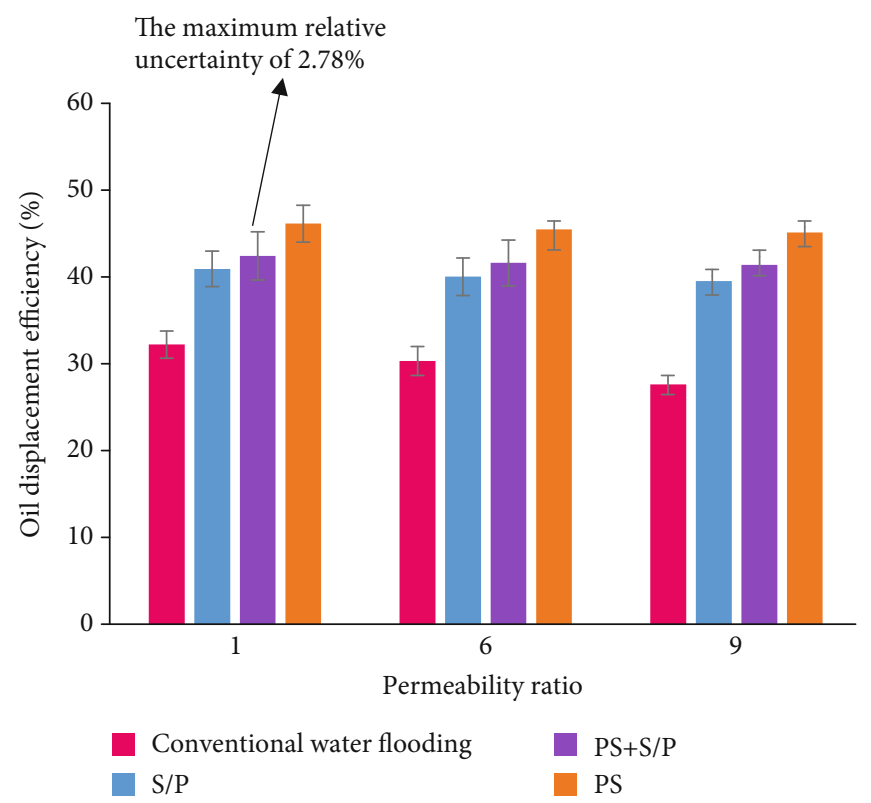

FIGURE 7: Oil displacement efficiency after different oil displacement measures.

relatively high under the condition of low concentration, which can better block high permeability areas; in addition, polymeric surfactant has good emulsifying and viscoelasticity properties, so it can further improve sweep volume and reduce the saturation of residual oil. The oil displacement effect of polymeric surfactant is better than that of polymeric surfactant with subsequent binary flooding. This is because when binary flooding is performed after the polymeric surfactant, the viscosity of the composite system decreases and the plugging effect on high permeability areas is poor. On the contrary, the injection of the binary system reduces the interfacial tension, so its displacement efficiency is better than that of single binary flooding.

Under the same oil displacement measures, with the increase of the permeability ratio, improved recovery efficiency increases and the variation in the values of various residual oil saturation gradually increases, which shows that injection of the displacement agent can effectively improve reservoir heterogeneity. From Figure 8, we can see that under the same permeability ratio, compared with conventional water flooding, all kinds of residual oil saturation are reduced.
Binary flooding mainly activates cluster and oil drop residual oils; polymeric surfactant with binary flooding mainly activates cluster, oil film, and column residual oils; polymeric surfactant flooding mainly activates cluster, oil drop, and column residual oils.

\subsection{Displacement Effect of Different Oil Displacement} Measures Using an Artificial Core. In order to explore the reliability of the microscopic model test results, the artificial core displacement tests were carried out in this paper. Using a core with a permeability ratio of 6 to explore displacement effect under different displacement measures, as shown in Figure 9, it can be seen from the recovery ratio and water cut curves that the water cut reduction of polymeric surfactant flooding is the largest, which has the best displacement effect, followed by polymeric surfactant with binary flooding and binary flooding alone; it is consistent with the oil displacement experiment results of the photoetched glass model; the corresponding recovery ratios were increased by $20.22 \%$, $16.30 \%$, and $13.09 \%$, respectively. 


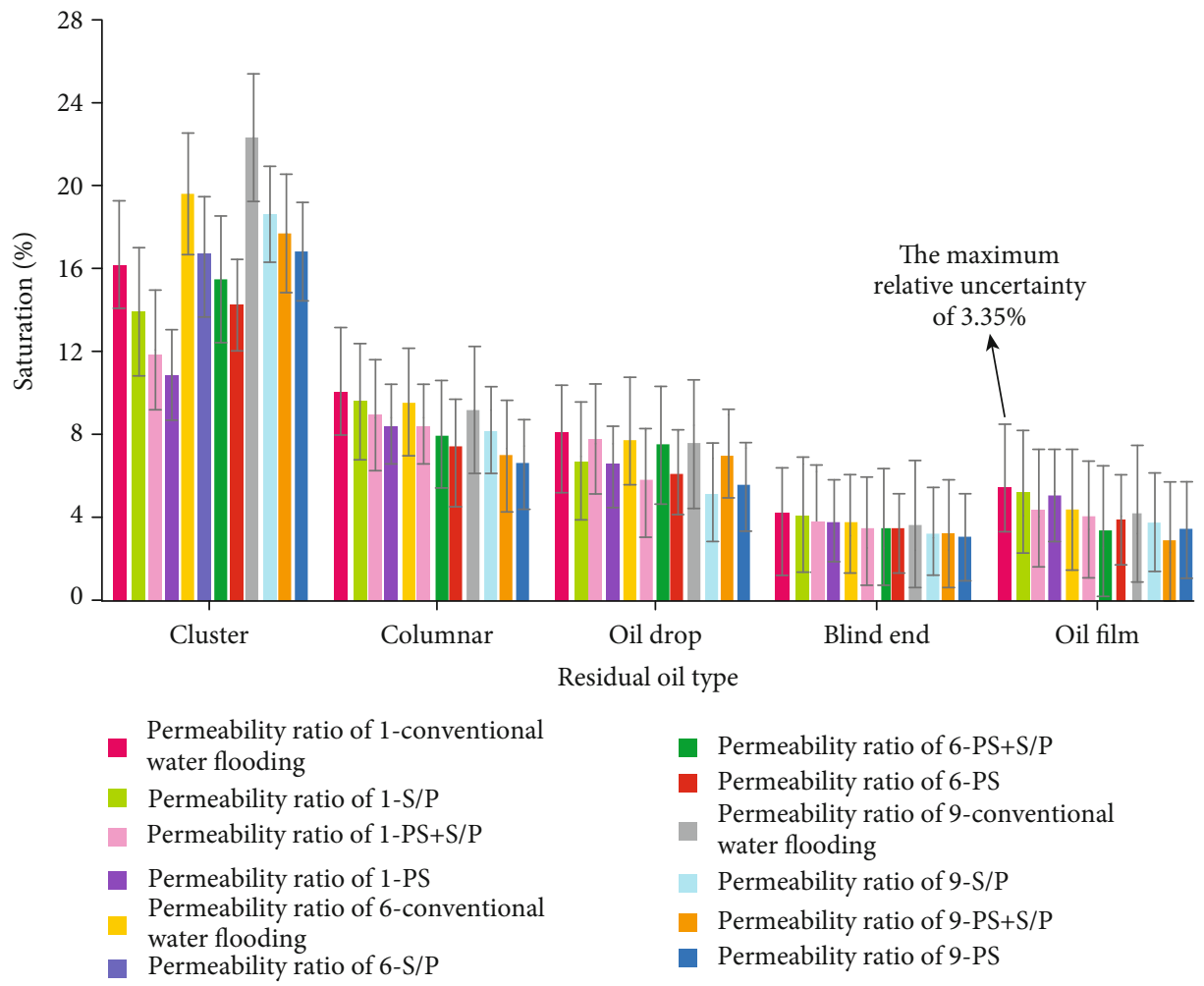

Figure 8: Saturations of various residual oils after different oil displacement measures.

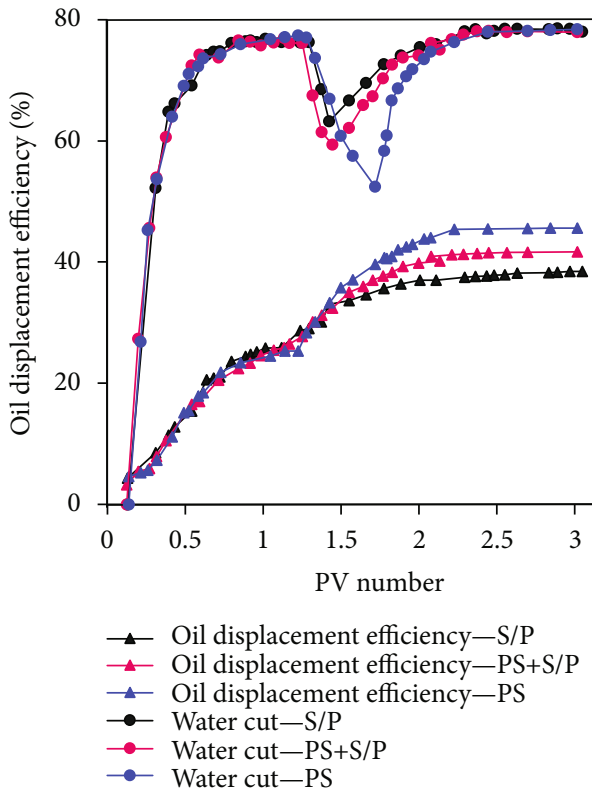

(a)

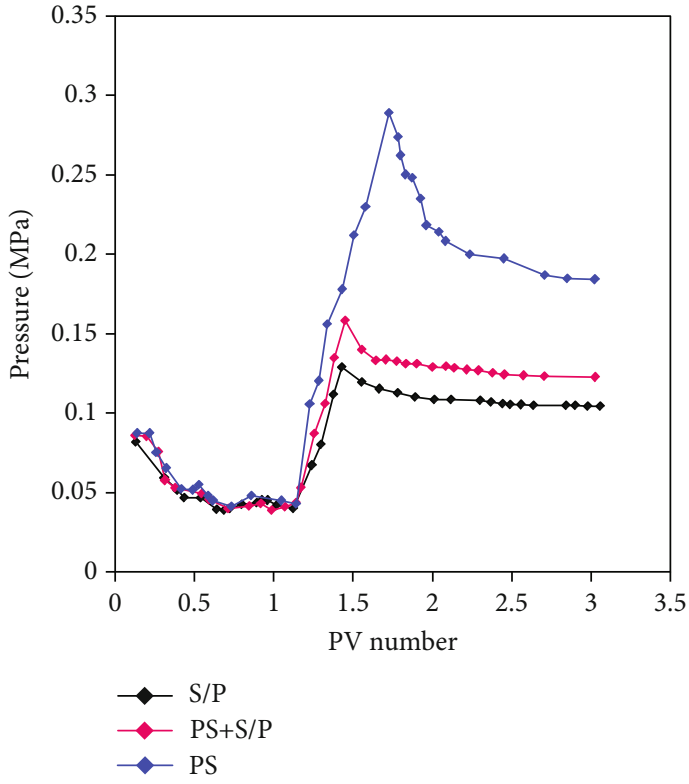

(b)

Figure 9: Curves of injection pore volume versus oil displacement efficiency, water cut, and pressure with a permeability ratio of 6 for different oil displacement measures: (a) oil displacement efficiency and water cut; (b) pressure.

From the injection pressure curve, as shown in Figure 9(b), it can be seen that the injection pressure is small during water flooding. After injecting the chemical agents, the injection pressure increases rapidly, the polymeric surfactant flooding has the highest injection pressure compared with other two oil displacement systems, and the subsequent water flooding 


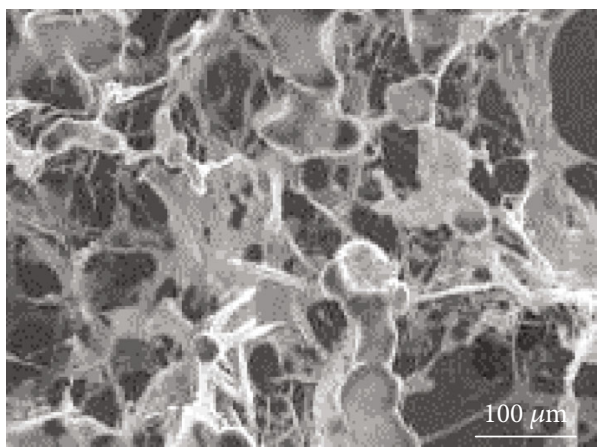

(a)

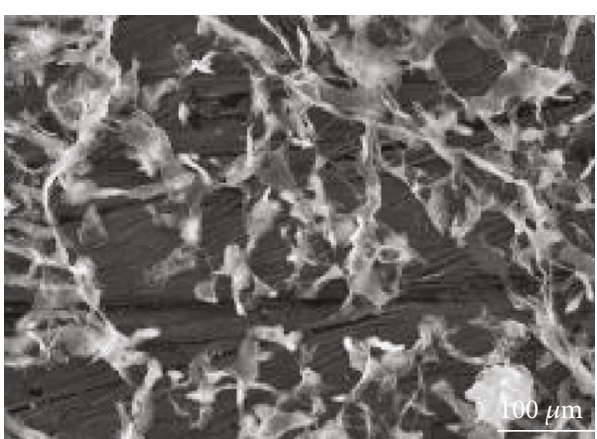

(b)

FIGURE 10: Microphotographs of the polymeric surfactant before and after the addition of a binary composite system: (a) PS, Cp (600 mg/L), 500 times magnification; (b) PS+S/P (polymer $1000 \mathrm{mg} / \mathrm{L}+0.3 \%$ surfactant), 500 times magnification.

stage can maintain a higher injection pressure. Polymeric surfactant with high viscosity can block the main channel in the high permeability layers, resulting in a sudden pressure rise and a sharp increase in the liquid absorption in the low permeability layers, and the residual oil begins to flow. In the actual field application, the polymeric surfactant is a shear diluting fluid, although the initial viscosity is very high, but the flow velocity near the embrasure is very high, so the actual viscosity is very low; therefore, it is easy to inject into the deep part of the reservoir. As the flow velocity decreases, the viscosity gradually increases, which helps to reduce the water-oil mobility ratio and expand the swept volume.

Polymeric surfactant flooding is better than polymeric surfactant and binary flooding system. This is because under the condition of the same treatment design and cost, polymeric surfactant flooding has a large injection PV. In addition, by comparing the scanning electron microscope analysis results as shown in Figure 10, for polymeric surfactant and the subsequent binary flooding system, we can see that the microscopic morphology of the polymeric surfactant before injection of binary flooding is a compact spatial network structure, so the viscosity is high. The addition of the binary system loosens the connection between the molecular aggregates which leads to a fracture phenomenon, which indicates that the addition of the binary system destroys the microcrosslinking structure of the polymeric surfactant and reduces the size of the molecular aggregates, thus greatly reducing the viscosity. However, polymeric surfactant with subsequent binary flooding are better than binary flooding alone because the polymeric surfactant and subsequent binary flooding system gives full advantages of the two chemical flooding systems, the injection of polymeric surfactant can further expand the swept volume of the low permeability region and the subsequent binary flooding can reduce oil-water interfacial tension and promote crude oil emulsification.

Maximum oil displacement efficiency is achieved through polymeric surfactant injection in the above experiments, so polymeric surfactant is selected to carry out further displacement experiments to investigate the influence of the permeability ratio on the displacement phenomenon. From Figure 11, it can be seen that, with the increase of the model permeability ratio, on the basis of conventional water flooding, for the models with permeability ratios of 1 , 6 , and 9 , the recovery ratio of polymeric surfactant flooding is increased by $19.52 \%, 20.22 \%$, and $21.47 \%$, respectively. From the injection pressure curve, it can be observed that the larger the model permeability ratio, the higher the injection pressure during water flooding. After polymer surfactant is injected, the injection pressure rises rapidly, the swept volume of the core increases, and the recovery ratio is enhanced. The porous medium is treated as multiple bundles of capillaries with equal lengths. For heterogeneous models with different permeability ratios, it is simplified to two capillary bundles with different radii. According to the Kozeny equation (equation (2)), permeability is proportional to the square of the circular tube radius and the permeability ratio is $K_{\max } / K_{\min }=\left(r_{\max } / r_{\min }\right)^{2}$. In addition, flow rate of the fluid passing through two capillary tubes can be calculated by Poiseuille's formula (equation (3)), which is expressed by $Q_{\max }$ and $Q_{\min }$. Assuming that the pressure difference $\Delta p$ at both ends of the two circular tubes is the same and the fluid viscosity $\mu$ and the length of the circular tubes $L$ are both equal, the flow rate ratio through the two circular tubes is $Q_{\max } / Q_{\min }=\left(K_{\max } / K_{\min }\right)^{2}$.

$$
\begin{aligned}
& K=\frac{\phi r^{2}}{8}, \\
& Q=\frac{\pi r^{4} \Delta p}{\mu L},
\end{aligned}
$$

where $K_{\max }$ is the permeability of the high permeability layer, $K_{\min }$ is the permeability of the low permeability layer, $r$ is the circular tube radius, $Q$ is the flow rate of the fluid, and $\varphi$ is porosity.

For heterogeneous models with a permeability ratio of 6 , the flow ratio of the high permeability layer to the low permeability layer is 36 ; for heterogeneous models with a permeability ratio of 9 , the flow ratio is 81 . The above analysis explains the phenomenon of injected water distribution in heterogeneous models with different permeability ratios. 


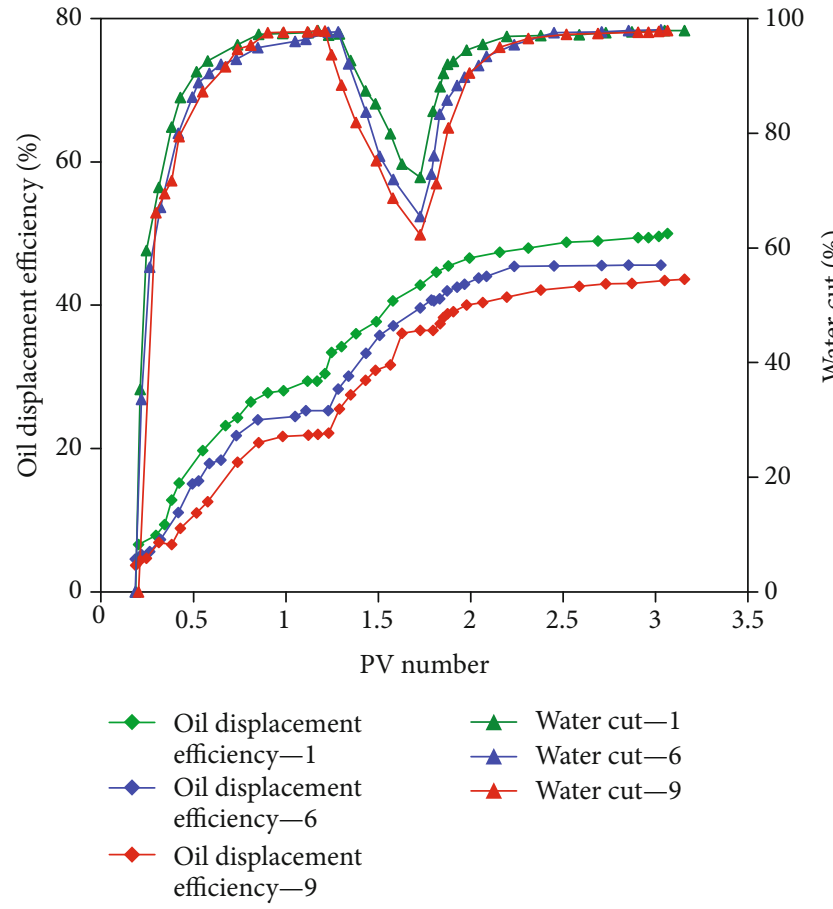

(a)

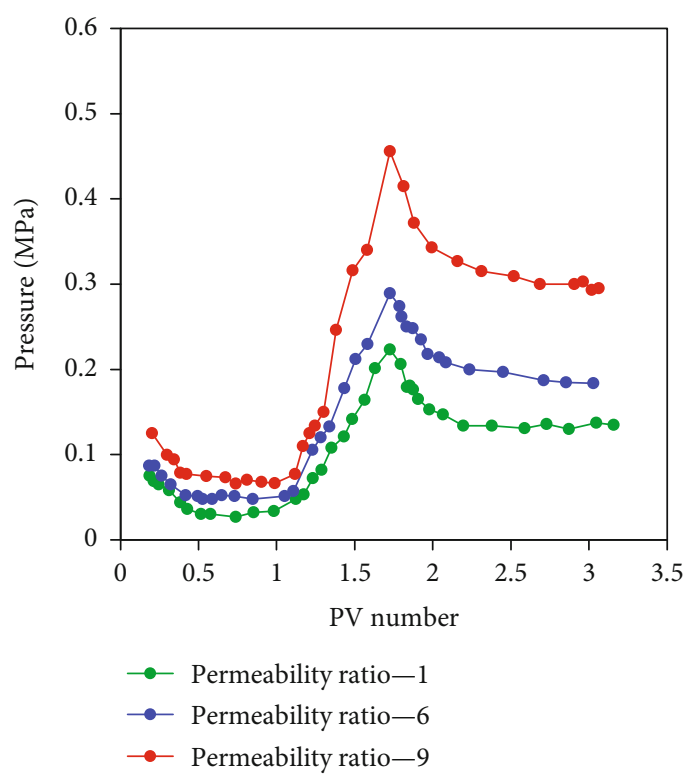

(b)

FIGURE 11: Curves of injection pore volume versus oil displacement efficiency under different permeability ratios for the polymeric surfactant: (a) oil displacement efficiency and water cut; (b) pressure.
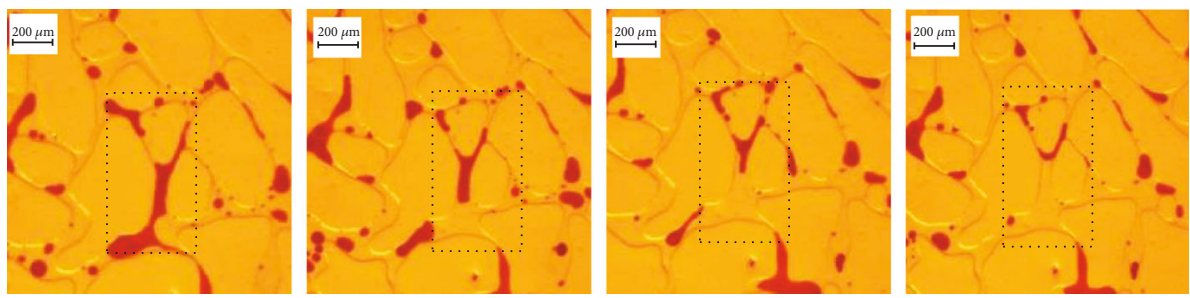

FIGURE 12: Schematic diagram of the displacement process of cluster residual oil (oil in pores is indicated by the red color).

\subsection{Displacement Mechanism of Polymeric Surfactant Flooding on Various Residual Oils}

3.3.1. Clustered Residual Oil. There are two formation mechanisms of cluster residual oil: one is that the small pores encompass the large pores-water always travels along the pores with low resistance during the displacement process, so the crude oil in one or a group of large pores surrounded by the small pores is difficult to be displaced, and the crude oil will remain in the large pores. The other is the formation of cluster residual oil for the small throat surrounded by the unobstructed large channel. Since water mainly flows along the large channel, then gathers in small channels, it will form clusters of residual oil. The cluster residual oil does not have a characteristic shape, it may be $\mathrm{Y}$ - or $\mathrm{H}$-shaped composed of several columnar residual oils, or it may be a large irregularly shaped residual oil formed by smaller clumps of residual oil. This kind of residual oil is small in quantity but exists in sections and therefore contains a large amount of oil.
From Figure 12, we can see that when the polymeric surfactant is injected into the pores, due to the increased viscosity of the injection fluid, it can spread to areas that cannot be reached by water flooding. During the displacement process, the residual oil first enters the large pores and forms an oil wall. This results in the increase of displacement resistance leading to increase of the swept volume of small pore channels. Injection of polymeric surfactant increases the displacement pressure difference at both ends of the pores, which results in the formation of a continuous channel between small pores and large pores, and eventually, the cluster residual oil is gradually converted into other types of residual oils and transported to the outlet end with the displacement fluid.

3.3.2. Oil Drop Residual Oil. After water flooding, there are some oil droplets that are separated from each other in the water phase. These oil droplets are stripped from the pore wall and subjected to the pore Jamin effect during flow, which cannot be displaced by water. Some throat radii are smaller 

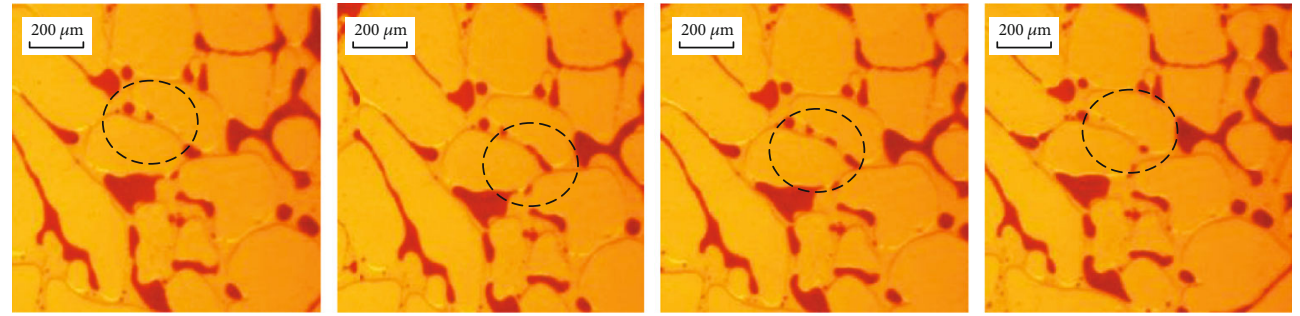

FIGURE 13: Schematic diagram of the displacement process of oil drop residual oil (oil in pores is indicated by the red color).
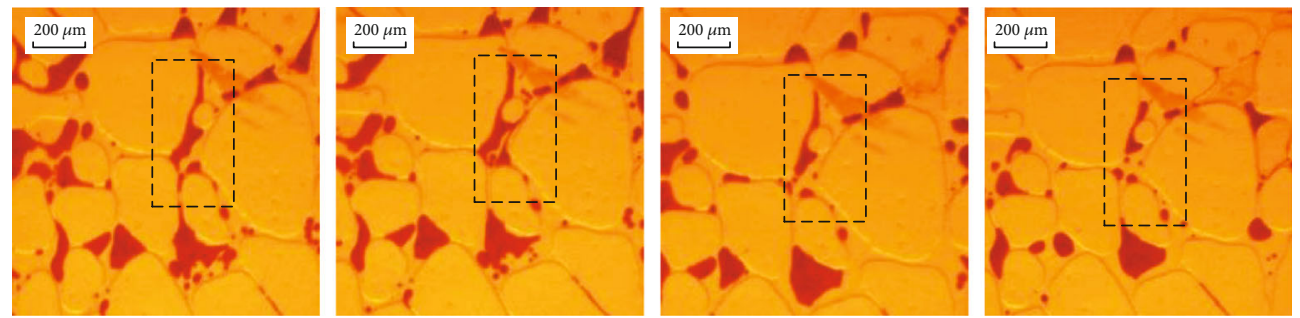

FIGURE 14: Schematic diagram of the displacement process of oil film residual oil (oil in pores is indicated by the red color).

than the radius of oil droplets, and oil droplets need to be deformed when passing through these throats. Due to the high interfacial tension between the oil and water, and small displacement pressure difference at both ends of the throat which is not enough to overcome the capillary force, the oil drops stay at the throat. As can be seen from Figure 13, after injecting the polymeric surfactant, oil droplets are elongated and broken under shearing action, then dispersed into small droplets. These oil droplets deform as they pass through the throat and appear like dumbbells; consequently, the resistance of oil drops through the small throat is reduced. In addition, the crude oil in the model is emulsified and transported to the outlet end in the form of oil in water under the action of the displacement liquid.

3.3.3. Oil Film Residual Oil. The oil-wet pore wall has a strong adhesion effect on the oil. After water flooding, the displacing force of water is less than the adhesive force of oil on the pore wall, water passes through the middle of the pores, and oil is adsorbed on the pore wall to form oil film residual oil. There is a large amount of residual oil in the oil-wet pores; oil film residual oil often serves as a channel connecting two independent oil bodies. As can be noticed from Figure 14, after injecting the polymeric surfactant, under a certain velocity gradient, the displacement front of the oil film lengthens. As a result, the oil film becomes thinner until it is completely carried down in the continuous process and droplets are emulsified in the form of oil-in-water emulsion. With the increase of shear stress, the polymeric surfactant can displace oil film residual oil. Due to the tensile stress caused by polymer macromolecules in the pore throat, the local pressure gradient increases, which is conducive to driving the residual oil in the pore throat.

The polymeric surfactant solution is a viscoelastic fluid which plays an important role in improving oil-water mobility and exhibits a normal stress in motion. The magnitude of normal stress is related to the degree of elasticity of the viscoelastic fluid and relative motion. The driving force of polymeric surfactant to oil film on the pore wall is greater than that of water flooding. On the other hand, the interfacial viscosity of the polymeric surfactant solution and oil is much higher than that of water and oil. The shear stress at the interface between the surfactant solution and the oil is proportional to the interfacial viscosity. It is a pulling force on the oil film in the shearing motion direction of the polymeric surfactant solution. Due to the effect of these two forces, the flow of residual oil which was immobile during water flooding is initiated, which is also a reason why polymeric surfactant flooding can improve the oil displacement efficiency.

3.3.4. Columnar Residual Oil. Due to the small throat radius in low permeability reservoirs and according to the capillary resistance calculation formula, $\mathrm{Pc}=2 \delta / r$ (Pc is capillary force, $\delta$ is the oil-water interfacial tension, and $r$ is the capillary radius), residual oil in some slender throats is subjected to very large capillary resistance. The displacement pressure is smaller than the capillary resistance, which causes the formation of columnar residual oil due to snap-off effect during the flow process. Due to the small seepage resistance and the relatively weak shearing action of the large pore channel for the polymeric surfactant flooding, the large pore channel can be effectively blocked. It leads to increased displacement pressure difference between the two ends of the small pore channels, resulting in more residual oil displacement and recovery. From Figure 15, we can see that the injection of the polymeric surfactant solution plays an emulsifying role in the columnar residual oil. The columnar residual oil deforms under the influence of displacement fluid and moves forward along the pore wall in a wire-drawing state. The columnar residual oil accumulates at the edge of the pore, which is then displaced by the displacement fluid. 

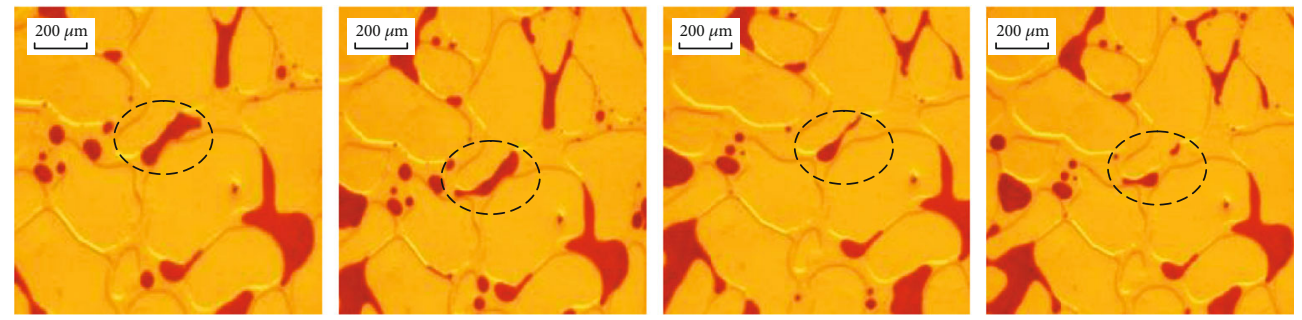

FIGURE 15: Schematic diagram of the displacement process of columnar residual oil (oil in pores is indicated by the red color).
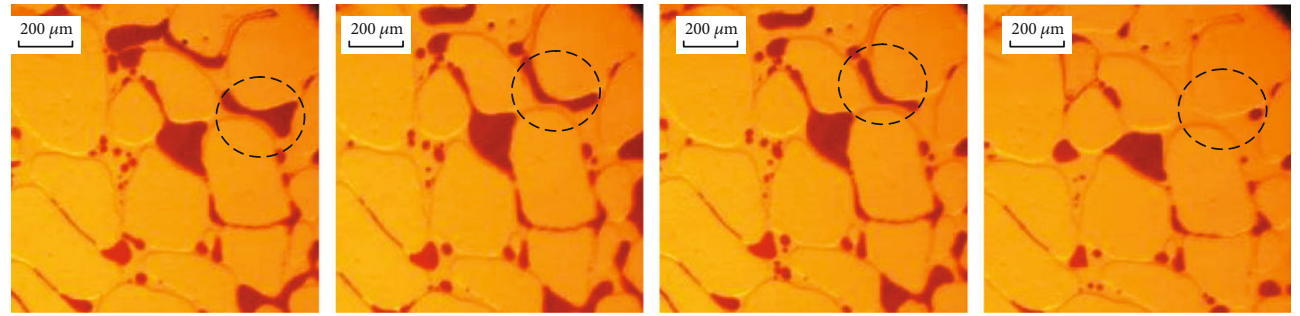

FIGURE 16: Schematic diagram of the displacement process of blind end residual oil (oil in pores is indicated by the red color).

3.3.5. Blind End Residual Oil. After water flooding, the residual oil at the blind end gathers in the pores closed at one end and is difficult to be displaced. The injection of the polymeric surfactant solution increases the shear force and has a pulling effect on the residual oil around the blind end pores, forming a continuous oil flow channel with the pore wall at the blind end. As can be seen from Figure 16, under the emulsification of polymeric surfactant, the blind end residual oil is eventually dispersed into droplets and displaced. Based upon fundamentals of fluid mechanics, the reservoir is composed of a large number of pore channels with complex geometric shapes, in which secondary flow is often induced and vortex is generated. In the process of two-phase displacement flow in reservoir pores, the size, intensity, and sweep area of the vortex play a very important role in the displacement of residual oil. Vortex phenomenon was also observed in the experiment. The ability of polymeric surfactant solution to displace residual oil mainly depends on its acting force on the crude oil and carrying capacity.

Therefore, during the displacement process in reservoir pores, the stress field formed by the viscoelastic vortex can be used as an index to measure the displacement ability of the polymeric surfactant solution to blind end residual oil. Only when the stress at the interface between the displacement fluid and the crude oil is sufficient to overcome the viscous forces and structural forces inside the crude oil does it become possible to drive the blind end residual oil. For the oil-wet blind end, the oil displacement efficiency of the blind end residual oil increases with the increase of the elasticity of the polymeric surfactant solution. For the water-wet blind end, the polymeric surfactant solution cannot improve the displacement efficiency of immovable residual oil. If the residual oil at the blind end is movable oil, or if other oil drops flow into the blind end and coalesce with the residual oil at the blind end, larger movable oil drops can be formed and displaced by viscoelastic fluid, which can reduce the blind end residual oil saturation.

\section{Conclusion}

(1) With the increase in the permeability ratio, the oil displacement efficiency of water flooding gradually decreases. In comparison with conventional water flooding, improved recovery efficiency and gradual reduction in the saturations of various residual oils after injecting different displacement schemes show that polymeric surfactant injection can effectively reduce the effect of reservoir heterogeneity on recovery

(2) Judging from the changes in residual oil saturation, binary flooding mainly activates cluster and oil drop residual oils; polymeric surfactant with binary flooding mainly activates cluster, oil film, and column residual oils; polymeric surfactant flooding mainly activates cluster, oil drop, and column residual oils

(3) From core flooding experiment results, under the condition of the same permeability ratio of 6 , polymeric surfactant flooding has the best displacement efficiency, followed by polymeric surfactant with binary system and the binary flooding system having the least efficiency. The corresponding recovery ratios were increased by $20.22 \%, 16.30 \%$, and $13.09 \%$, respectively. After injecting the binary flooding system, the microcrosslinking structure of the polymeric surfactant is destroyed, which reduces the size of the molecular aggregates. This greatly reduces 
the viscosity and weakens the plugging ability of the polymeric surfactant. Due to the excellent viscoelasticity and emulsification characteristics of the polymeric surfactant, the residual oil is displaced by pulling and deformation

\section{Data Availability}

The data in this article are all available.

\section{Conflicts of Interest}

The authors declare that they have no conflicts of interest.

\section{Acknowledgments}

This work was financially supported by the National Natural Science Foundation of China (51574085) and the National Natural Science Foundation of Heilongjiang Province (E2016008).

\section{References}

[1] A. Rostami, A. Baghban, A. H. Mohammadi, A. HemmatiSarapardeh, and H. Habibzadeh, "Rigorous prognostication of permeability of heterogeneous carbonate oil reservoirs: smart modeling and correlation development," Fuel, vol. 236, pp. 110-123, 2019.

[2] G. Javanbakht, M. Arshadi, T. Qin, and L. Goual, "Micro-scale displacement of NAPL by surfactant and microemulsion in heterogeneous porous media," Advances in Water Resources, vol. 105, pp. 173-187, 2017.

[3] L. Yu, M. Z. Dong, B. X. Ding, and Y. G. Yuan, "Experimental study on the effect of interfacial tension on the conformance control of oil-in-water emulsions in heterogeneous oil sands reservoirs," Chemical Engineering Science, vol. 189, pp. 165178, 2018.

[4] H. Liu, Y. Zhang, Y. Li, J. Hou, and Y. Liu, "Influence on emulsification in binary flooding of oil displacement effect," Journal of Dispersion Science and Technology, vol. 37, no. 1, pp. 89-96, 2016.

[5] D. Y. Yin and X. R. Zhang, "Evaluation and research on performance of a blend surfactant system of alkyl polyglycoside in carbonate reservoir," Journal of Petroleum Science and Engineering, vol. 111, no. 111, pp. 153-158, 2013.

[6] L. D. Sun, X. L. Wu, W. F. Zhou, X. J. Li, and P. H. Han, "Technologies of enhancing oil recovery by chemical flooding in Daqing oilfield, NE China," Petroleum Exploration and Development, vol. 45, no. 4, pp. 673-684, 2018.

[7] S. Liu, A. Shen, F. Qiu, S. Liang, and F. Wang, "Matching relationship and alternating injection for polymer flooding in heterogeneous formations: a laboratory case study of Daqing oilfield," Energies, vol. 10, no. 7, p. 1018, 2017.

[8] H. Zhong, W. Zhang, J. Fu, J. Lu, and H. Yin, "The performance of polymer flooding in heterogeneous type II reservoirs-an experimental and field investigation," Energies, vol. 10, no. 4, p. 454, 2017.

[9] Z. Wang, X. Le, Y. Feng, and C. Zhang, "The role of matching relationship between polymer injection parameters and reservoirs in enhanced oil recovery," Journal of Petroleum Science and Engineering, vol. 111, no. 111, pp. 139-143, 2013.
[10] D. M. Wang, H. F. Xia, Z. C. Liu, and Q. Y. Yang, "Study of the mechanism of polymer solution with visco-elastic behavior increasing microscopic oil displacement efficiency and the forming of steady "Oil Thread" flow channels," in SPE Asia Pacific Oil and Gas Conference and Exhibition, Jakarta, Indonesia, 2001.

[11] W. Cao, K. Xie, X. Lu, Y. Liu, and Y. Zhang, "Effect of profilecontrol oil-displacement agent on increasing oil recovery and its mechanism," Fuel, vol. 237, pp. 1151-1160, 2019.

[12] A. M. AlSofi, J. X. Wang, and Z. F. Kaidar, "SmartWater synergy with chemical EOR: effects on polymer injectivity, retention and acceleration," Journal of Petroleum Science and Engineering, vol. 166, pp. 274-282, 2018.

[13] S. Al-Hajri, S. Mahmood, H. Abdulelah, and S. Akbari, “An overview on polymer retention in porous media," Energies, vol. 11, no. 10, p. 2751, 2018.

[14] E. Unsal, A. B. G. M. Ten Berge, and D. A. Z. Wever, "Low salinity polymer flooding: lower polymer retention and improved injectivity," Journal of Petroleum Science and Engineering, vol. 163, pp. 671-682, 2018.

[15] Q. Z. Li, W. F. Pu, B. Wei, F. Y. Jin, and K. X. Li, "Static adsorption and dynamic retention of an anti-salinity polymer in low permeability sandstone core," Journal of Applied Polymer Science, vol. 134, no. 8, 2017.

[16] W. T. Li, C. L. Dai, O. Y. Jian et al., "Adsorption and retention behaviors of heterogeneous combination flooding system composed of dispersed particle gel and surfactant," Colloids and Surfaces A: Physicochemical and Engineering Aspects, vol. 538, pp. 250-261, 2018.

[17] Z. W. Jia, Q. Y. Yang, M. Yuan, Z. Hou, and L. Zhang, "Experimental study on influential factors of ASP displacement efficiency in Daqing oilfield," Acta Petrolei Sinica, vol. 27, no. S1, pp. 102-105, 2006.

[18] M. Kazempour, E. J. Manrique, V. Alvarado, J. Zhang, and M. Lantz, "Role of active clays on alkaline-surfactant-polymer formulation performance in sandstone formations," Fuel, vol. 104, pp. 593-606, 2013.

[19] J. Ngimhuang, J. I. Furukawa, T. Satoh, T. Furuike, and N. Sakairi, "Synthesis of a novel polymeric surfactant by reductive $\mathrm{N}$-alkylation of chitosan with 3-O-dodecyl-D-glucose," Polymer, vol. 45, no. 3, pp. 837-841, 2004.

[20] T. del Castillo-Santaella, J. M. Peula-García, J. MaldonadoValderrama, and A. B. Jódar-Reyes, "Interaction of surfactant and protein at the $\mathrm{O} / \mathrm{W}$ interface and its effect on colloidal and biological properties of polymeric nanocarriers," Colloids and Surfaces B: Biointerfaces, vol. 173, pp. 295-302, 2019.

[21] D. T. Glatzhofer, G. Cho, C. L. Lai, E. A. O'Rear, and B. M. Fung, "Polymerization and copolymerization of sodium 10-undecen-1-yl sulfate in micelles and in admicelles on the surface of alumina," Langmuir, vol. 9, no. 11, pp. 2949-2954, 1993.

[22] D. Burgemeister, T. Farrell, and C. Schmidt, "Lyotropic lamellar polysiloxanes with isomeric amphiphilic side chains," Macromolecular Chemistry and Physics, vol. 207, no. 4, pp. 396-403, 2006.

[23] H. Sawada, A. Takebayashi, M. Uejima, and T. Murakami, "Preparation and applications of novel fluoroalkyl endcapped oligomeric nanocomposites," Polymers for Advanced Technologies, vol. 3, no. 1, pp. 46-65, 2012.

[24] Y. Ma, J. Hao, K. Zhao et al., "Biobased polymeric surfactant: natural glycyrrhizic acid-appended homopolymer with 
multiple pH-responsiveness," Journal of Colloid and Interface Science, vol. 541, pp. 93-100, 2019.

[25] M. Yue, W. Zhu, H. Han, H. Song, Y. Long, and Y. Lou, "Experimental research on remaining oil distribution and recovery performances after nano-micron polymer particles injection by direct visualization," Fuel, vol. 212, pp. 506-514, 2018.

[26] D. Wu, B. Ju, X. Chen, Y. Fu, and E. T. Brantson, "Experimental evaluation of different strategies for improving oil recovery at high-water-cut by quantitative etched-glass micro-models," Energy Exploration \& Exploitation, vol. 34, no. 4, pp. 586-605, 2016.

[27] Y. Fang, E. Yang, D. Yin, and Y. Gan, "Study on distribution characteristics of microscopic residual oil in low permeability reservoirs," Journal of Dispersion Science and Technology, pp. 1-10, 2019.

[28] W. Zhu, X. Xia, S. Guo, J. H. Li, Z. Song, and G. Qu, "Microscopic oil displacement mechanism of indigenous microorganisms under high temperature and high pressure conditions," Acta Petrolei Sinica, vol. 35, no. 3, pp. 528-535, 2014.

[29] Y. Gan, Q. Cheng, W. Sun, W. Gao, X. Liu, and Y. Liu, “The stability criterion model and stability analysis of waxy crude oil pipeline transportation system based on excess entropy production," Journal of Thermal Science, vol. 27, no. 6, pp. 541-554, 2018. 

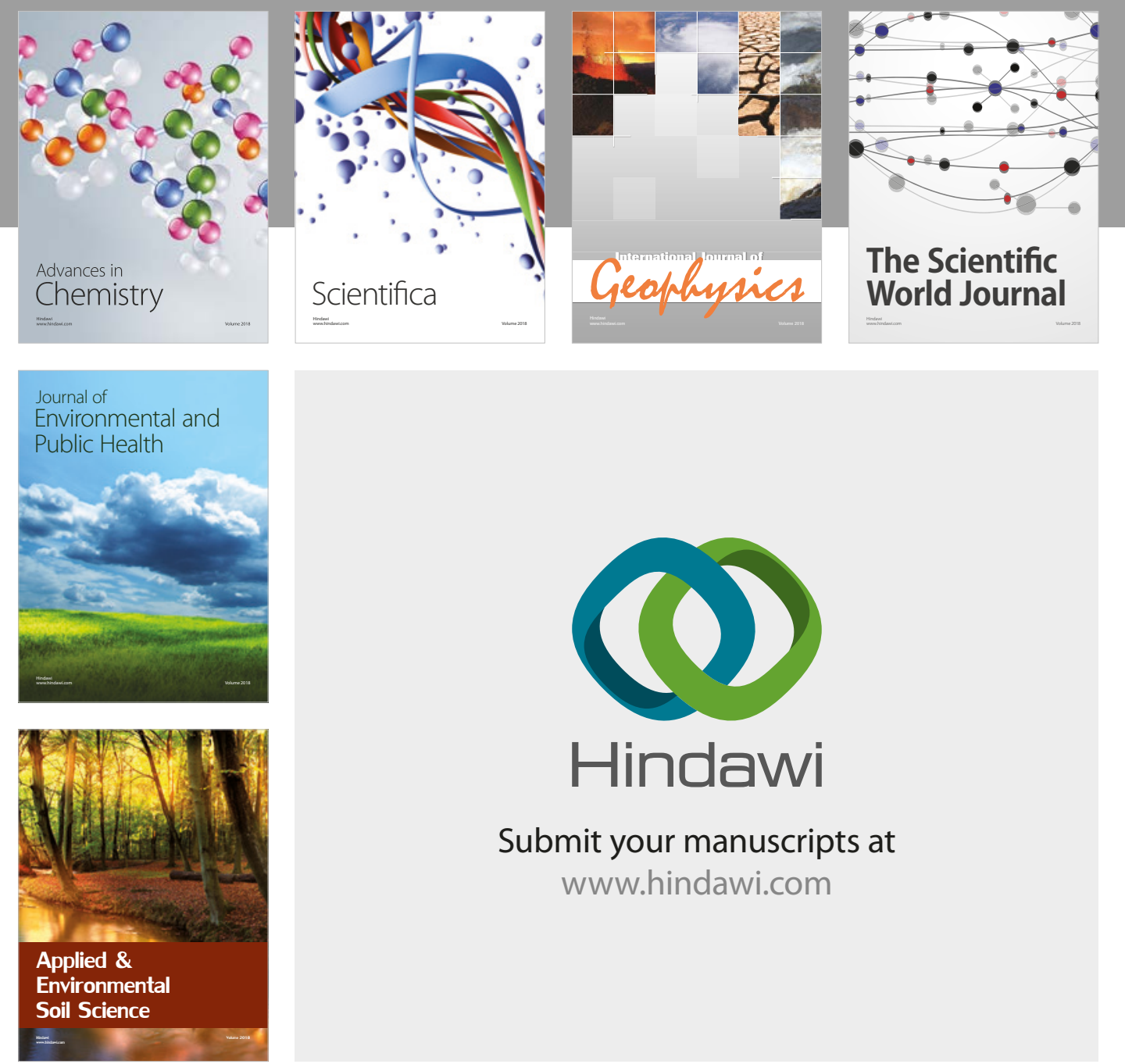

The Scientific

\section{World Journal}
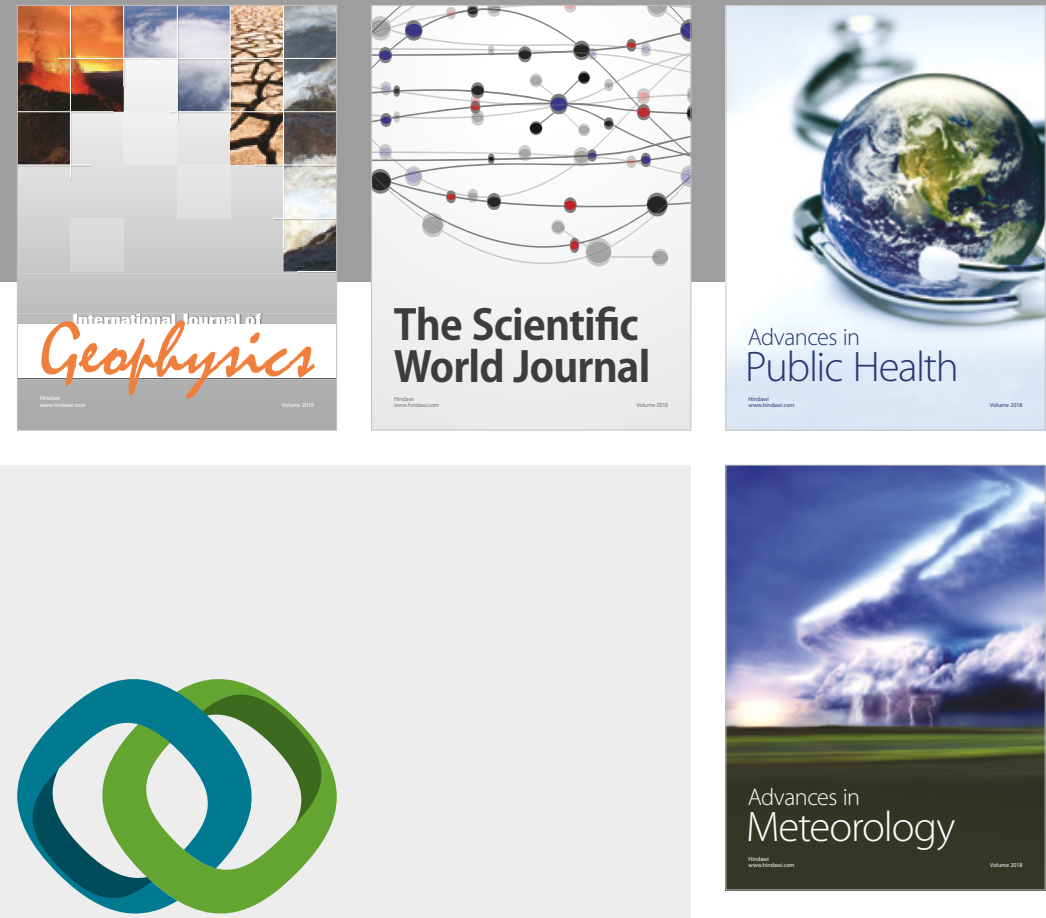

Advan

Public Health

\section{Hindawi}

Submit your manuscripts at

www.hindawi.com
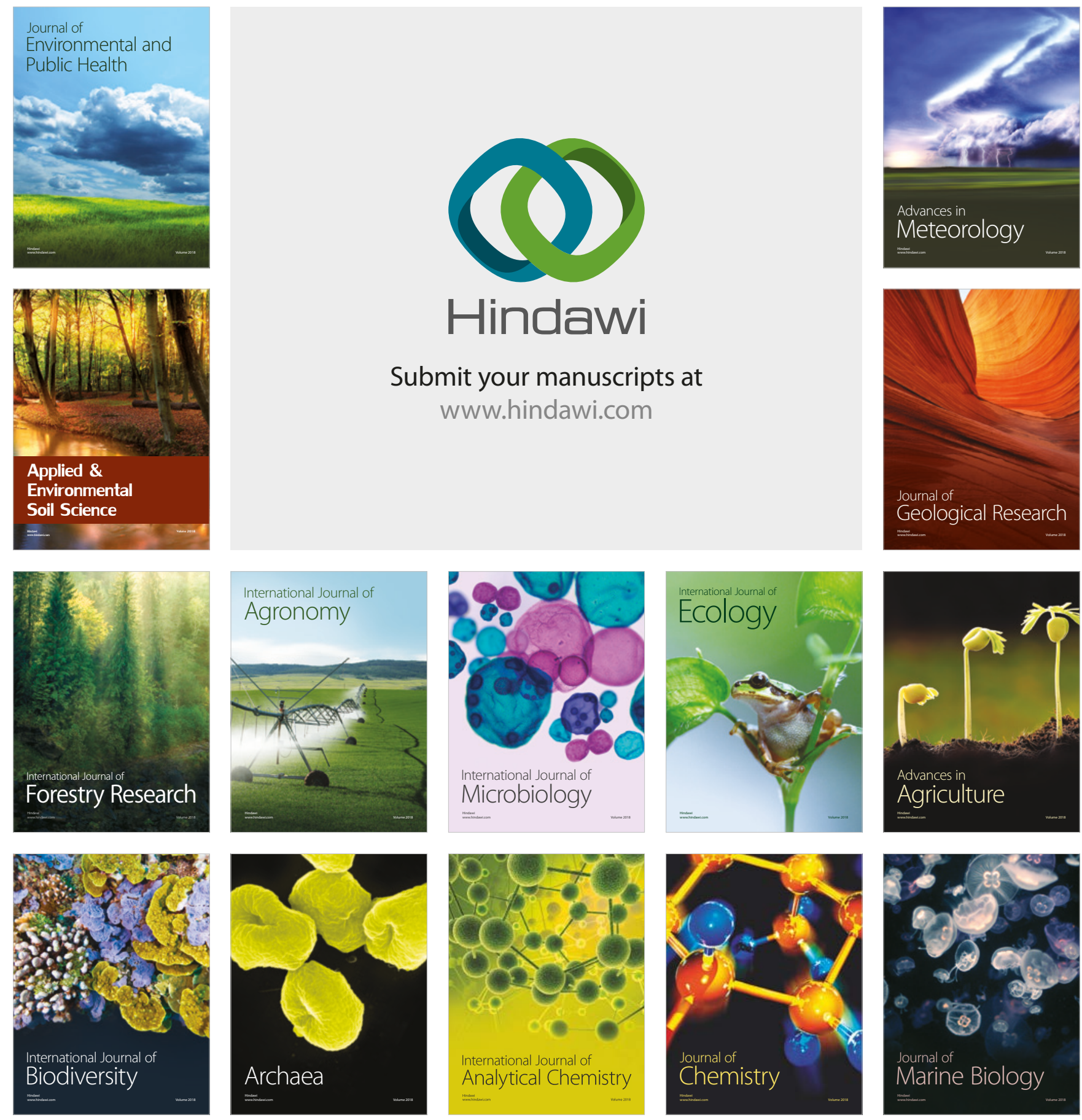